\title{
SECA E A SAÚDE DAS POPULAÇÕES RESIDENTES EM REGIÕES DA AMAZÔNIA BRASILEIRA NOS ANOS DE 2005, 2010 E 2015
}

\author{
GOMES, Ana Carla dos Santos - anacarlasg02@gmail.com \\ Universidade Federal do Oeste do Pará / UFOPA
}

COSTA, Gabriel Brito - gabrielbritocosta@gmail.com Universidade Federal do Oeste do Pará / UFOPA

\author{
SILVA, Julio Tóta da - totaju@gmail.com \\ Universidade Federal do Oeste do Pará / UFOPA
}

COUTINHO, Maytê Duarte Leal - mayte.coutinho@funceme.br

Fundação Cearense de Meteorologia e Recursos Hídricos / FUNCEME

COSTA, Micejane da Silva - micejane@yahoo.com.br Universidade Federal do Rio Grande do Norte / UFRN

FITZJARRALD, David Roy - dfitzjarrald@albany.edu University of Albany

\begin{abstract}
RESUMO: O objetivo deste trabalho é verificar os impactos ocasionados pelos três últimos eventos de seca na saúde da população de catorze municípios na Amazônia, agrupados em quatro grupos com características pluviométricas homogêneas. Utilizaramse dados diários e mensais de precipitação e temperatura do ar disponibilizado pelo Banco de Dados Meteorológicos para Ensino e Pesquisado Instituto Nacional de Meteorologiae de internações por doenças respiratóriasdo Sistema Único de Saúde (DATASUS), do período de 2000 a 2015. Levando-se em consideração as especificidades dos dados em estudo utilizou-se a modelagem via Equações de Estimação Generalizada para verificar associações significativas e o risco relativo de aumento da ocorrência das internações da população exposta a ausência de chuva. Os resultados mostraram que as estações influenciadas pela seca de 2005 estão localizadas a sudoeste da Amazônia, as mesmas foram pouco afetadas pelo evento de 2015. Embora o evento de seca de 2010 tenha sido mais extenso espacialmente do que 2005, o efeito da diminuição das chuvas a partir dos dados observados de um evento para o outro só se mostrou perceptível nas estações localizadas na região central da Amazônia. Já a seca de 2015 teve maior impacto nos municípios em que as estações estão localizadas ao norte e nordeste da Amazônia. As populações consideradas mais vulneráveis por meio dos riscos captados pelo modelo foram as de Itaituba, Monte Alegre e Porto de Moz. Conclui-se que ao comparar as regiões percebe-se que os três eventos de seca afetam de forma diferente os quatros grupos, o que provavelmente está associado ao efeito diferencial dos mecanismos causadores das secas nas diferentes áreas da Amazônia.
\end{abstract}

PALAVRAS-CHAVE: Doenças Respiratórias, Precipitação, Temperatura, Risco Relativo.

DROUGHT AND HEALTH OF RESIDENT POPULATIONS IN BRAZILIAN AMAZON REGIONS IN THE YEARS OF 2005, 2010 AND 2015

ABSTRACT: The objective of this work is verify the impacts occasioned due to the last three dry events on population heath of fourteen counties in Amazon, grouped in four groups with homogeneous rainfall characteristics. Was used daily and monthly data of rainfall and air temperature available in the Meteorological Data Set for Studies and Research of National Institute of Meteorology and about hospital internment referent to respiratory diseases by Heath Unique system (DATASUS), of the period from 2000 to 
2015. Taking in consideration the specificity of the data in study was used modeling by way of Generalized Estimation Equations to verify significant associations and the relative risk of increase of the internment occurrence expose to absence of rainfall. The results showed seasons influenced by the 2005 drought are located in the Amazonia southwest, but were not affected by the event in 2015. Although the 2010 drought was more extensive spatially than 2005, the effect rainfall decreasing from observed data between events was only perceptible in central Amazonia region stations. The 2015 drought had greater impact in the municipalities where the stations are located in the north and northeast of the Amazonia. The population considered more vulnerable by means of the risks captured by the model were Itaituba, Monte Alegre and Porto de Moz. In summary comparing the regions its perceive that the 3 events of drought affected on different ways the four groups, what probably is associate to the differential effect of mechanisms of drought former in the different areas of the Amazon.

KEYWORDS Respiratory Disease, Rainfall, Temperature, Relative Risk

\section{INTRODUÇÃO}

A região da Amazônia com uma área de aproximadamente 5.217,423 $\mathrm{km}^{2}$ ocupa $61 \%$ do território Brasileiro (IBGE, 2010). Devido a sua grande extensão territorial esta região apresenta diferentes características meteorológicas e climáticas (Santos et al., 2014). Essas diferenças estão relacionadas a efeitos topográficos e à presença de sistemas meteorológicos que atuam em diferentes escalas de tempo e são modulados por mecanismos de interação na interface oceano-atmosfera, que podem promover totais pluviométricos acima e/ou abaixo da média climatológica (De Souza et al., 2005). O principal mecanismo tropical de circulação oceano-atmosfera que modula a precipitação nessa região é o El Niño Oscilação Sul (ENOS) sobre o Oceano Pacífico e o gradiente meridional inter-hemisférico de anomalias de temperatura da superfície do mar (TSM) sobre o oceano Atlântico (Nobre e Shukla, 1996; De Souza et al., 2000), atua em suas diferentes fases favorecendo ou desfavorecendo a atividade convectiva em áreas tropicais. Logo, diversos eventos de precipitação extrema estão associados às anomalias de TSM dos oceanos Pacífico e Atlântico (Marengo et al., 2008a; Marengo et al., 2008b; Marengo, 2011; Vale et al., 2011; Sena et al., 2012; Coelho et al., 2012, Santos et al., 2012). Ainda, segundo o Intergovernmental Panel on Climate Change IPCC (2007), as chuvas na Amazônia apresentam variabilidades interanual e em escala de tempo interdecadal, que são mais importantes que tendências de aumento ou redução. Estas variabilidades estão associadas a padrões de variação nas mesmas escalas de tempo nos oceanos Pacifico e Atlântico, com a variabilidade interanual associada ao ENOS, ou à Oscilação do Atlântico Norte (North Atlantic Oscillation - NAO), e a variabilidade decadal com a Oscilação Decadal do Pacifico (Pacific Decadal Oscillation - PDO), no Pacífico, e com a Oscilação Multidecadal do Atlântico (Atlantic Mutidecadal Oscillation - AMO), no Atlântico.

Marengo et al. (2008) afirmavam que em 2005, uma grande parte da bacia amazônica ocidental experimentou a seca mais grave dos últimos 40 anos e uma das mais intensas dos últimos cem anos. O quinto relatório do IPCC (2013) afirma que eventos extremos, como as secas que haviam ocorrido nos anos de 2005 e de 2010 no oeste e sudoeste da Amazônia, num cenário futuro de aumento de $\mathrm{CO}_{2}$ e diminuição de aerossóis, provavelmente se tornariam mais frequentes. Em 2015, após a divulgação do quinto relatório do IPCC a região amazônica voltou novamente a se deparar com um evento extremo de seca, com proporções maiores do que os últimos dois eventos de 2005 e 2010. O 
evento de 2015 foi relacionado ao fenômeno El Niño, a Agência Meteorológica do Japão e a National Oceanic and Atmospheric Administration (NOAA) detectaram a formação do El Niño no fim do mês de maio/2015 com intensidade prevista para a categoria forte. No fim do mês de Junho/2015 a região central e leste do Pacífico alcançaram anomalias de temperatura equivalentes a El Niño em patamar forte, confirmando a previsão. Destaca-se que no mês de Novembro/2015 as temperaturas foram superiores as registradas no grande evento de 1997/98 e que o fenômeno se estendeu até a metade do ano de 2016 na categoria forte ao longo de todos os meses de 2015. A Organização meteorológica mundial (OMM) declarou que o evento foi um dos três maiores já registrados, juntamente com 1982/83 e 1997/98.

A estiagem em 2015 na região da Amazônia Central foi agravada pelo fenômeno El Niño, o que provocou diminuição do nível do Rio Negro (Amazonas) de mais de sete metros somente no mês de outubro/2015 e a capital do estado Amazonas, Manaus, ficou por mais de 20 dias seguidos, coberta por fumaça proveniente de mais de $11 \mathrm{mil}$ focos de queimadas segundo o Instituto Nacional de Pesquisas Espaciais (INPE, 2016). Municípios paraenses como Altamira, Belterra, Monte Alegre, Óbidos e Santarém não tiveram estações chuvosas em 2015 (INMET, 2016). As chuvas ficaram até 50\% abaixo da média em BelémPA, onde as tradicionais chuvas vespertinas não ocorreram e os reservatórios das usinas hidroelétricas do estado do Pará operaram com níveis muito baixos (SIPAM, 2016).

Os impactos diretos e indiretos ocasionados pela ausência de chuva são preocupantes, pois afetam diversos setores ligados à sociedade como a agropecuária, economia, indústria, navegação, comprometimento da saúde humana etc. Vários tipos de doenças são influenciados por um clima mais quente, a literatura destaca as doenças respiratórias e as cardiovasculares (Gomes et al., 2013). As doenças respiratórias (DR) possuem posição de destaque no Brasil, de acordo com o último consenso brasileiro (Solé et al., 2006), a rinite é descrita na literatura como uma das doenças crônicas mais frequentes na infância. Embora insuficientemente estimada, traz desconforto e pode associar-se a problemas graves como apneia do sono, asma e infecções respiratórias repetidas (Sousa et al., 2012). A prevalência média de sintomas relacionados à rinite alérgica no Brasil, por exemplo, foi de $29,6 \%$ entre adolescentes (de 13 a 14 anos) e $25,7 \%$ entre escolares (de seis a sete anos) entre 2002 e 2003. Tanto sinusite quando rinite e outras doenças respiratórias consideradas bastante comuns na prática clínica podem significar diminuição da qualidade de vida, agravo de comorbidades e exigir significativos gastos com saúde (Souza et al. 2012). O Brasil está no grupo de países com as maiores prevalências de rinite alérgica no mundo (Solé et al., 2006). A prevalência tem aumentado ao longo dos anos e provavelmente é subestimada, pois muitos indivíduos não a reconhecem como uma doença e não procuram atendimento médico. Por outro lado, os profissionais de saúde frequentemente negligenciam a rinite. Ainda assim, a rinite alérgica encontra-se entre as dez razões mais frequentes para a procura de atendimento primário à saúde (Ibiapina et al., 2008).

Eventos extremos climáticos começam a ser mais frequentes, gerando preocupação crescente quanto aos potenciais efeitos à saúde humana, especialmente aqueles relacionados ao sistema respiratório (Mourão et. al., 2007). Alguns estudos têm mostrado a relação entre a variação sazonal e as 
proporções de atendimentos ambulatoriais por doenças respiratórias, assim como a ocorrência de internações hospitalares (Rosa et al., 2008a). Vale ressaltar que a redução da umidade relativa do ar a valores abaixo de $30 \%$ é considerada de risco para a integridade das vias aéreas (Mold, 2008), dificultando a homeostase interna do aparelho respiratório. Nos meses de chuva, em contraposição à problemática vivenciada nos meses de seca, a alta umidade relativa do ar, aliada ao maior tempo de permanência nos ambientes internos, ao menor arejamento e exposição ao sol dos espaços domiciliares, com consequente crescimento de mofo e fungos, são fatores que podem contribuir para o aumento das DR, especialmente as alérgicas (Mold, 2008).

Alguns vírus apresentam comportamento altamente sazonal, com maior frequência no período de chuvas de clima tropical, como o da Amazônia legal. Esses vírus são frequentemente causadores de doenças respiratórias, principalmente das vias aéreas superiores (Thomazelli et al., 2007). Entretanto, - conhecimento acerca do comportamento das DR em áreas de bioma amazônico e da possível influência dos ciclos típicos de seca e chuva sobre a saúde humana é escasso (Rosa et al., 2008b). O objetivo deste trabalho é verificar os impactos dos efeitos dos últimos três eventos de seca, ocorrido nos anos de 2005, 2010 e 2015 na região da Amazônia Brasileira, associando com dados de internações por doenças respiratórias.

\section{DADOS E METODOLOGIA}

\subsection{DADOS E ÁREA DE ESTUDO.}

Os dados de precipitação e temperatura média do ar foram obtidos do Banco de Dados Meteorológicos para Ensino e Pesquisa (BDMEP), do Instituto Nacional de Meteorologia - INMET, período de 2000 a 2015. As estações foram selecionadas seguindo as recomendações da Organização Mundial de Meteorologia (OMM), estabelecido no documento técnico no. 341. Este documento recomenda-se a (i) descartar todos os meses mostrando qualquer valor diário faltando e (ii) descarte de séries mensais com falhas de três ou mais meses consecutivos, ou mais de cinco meses alternativos faltando.

Para verificação dos impactos dos eventos de secas na saúde humana foram utilizados dados de hospitalizações por causas respiratórias, segundo local de internação para cada município em estudo, obtidos nos bancos de dados informatizados do Ministério da Saúde, através das Autorizações de Internações Hospitalares (AIH) do Sistema Único de Saúde (SUS) para o período de 2000 a 2015 (DATASUS, 2016).

Para representar a Amazônia Brasileira, foram utilizados dados de catorze municípios geograficamente distribuídos conforme Figura 1, (a escolha dos mesmos ocorreu para atender as normas citadas anteriormente da OMM). Sendo três do Estado do Acre (Cruzeiro do Sul, Rio Branco e Tarauacá), quatro do Estado do Amazonas (Eirunepé, Labréa, Manaus e Manicoré) seis do Estado do Pará (Altamira, Belterra, Itaituba, Monte Alegre, Óbidos e Porto de Moz) e um no Estado do Mato Grosso (Matupá). 


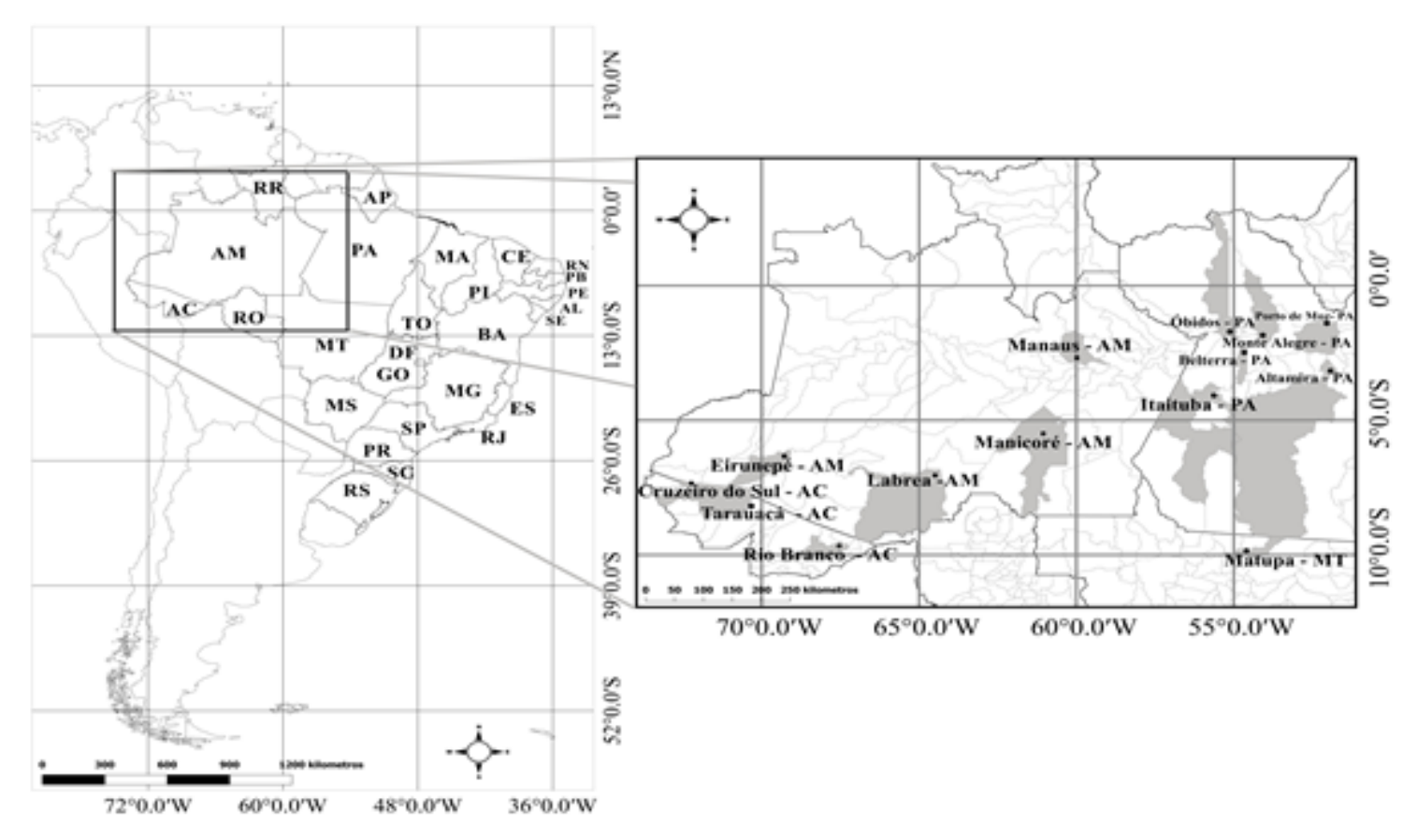

Figura 1 - Mapa de localização das estações em estudo na Amazônia Brasileira.

\subsection{MÉTODOS}

Para formação das regiões, agruparam-se os municípios onde foram encontradas variabilidades homogêneas de precipitação. Foram utilizados a análise de variância (ANOVA) para verificar a significância dos efeitos entre grupos em cada variável. Para captar associações significativas e o risco relativo influenciado pela ausência de chuva nas doenças respiratórias, foi empregada a modelagem via Equações de estimação generalizadas (Generalized Estimating Equations - GEE).

Segundo Gomes et al. (2015) a modelagem da relação clima e saúde, depara-se com dados correlacionados, ou apesar das variáveis estudadas serem independentes, a informação sobre esta(s) é coletada repetidas vezes ao longo do tempo, tornando as observações correlacionadas.

A modelagem via GEE foi proposta por Zeger e Liang (1986) e Liang e Zeger (1986) com o intuito de estimar parâmetros de regressão quando os dados estão correlacionados. Os autores basearam-se nos Modelos Lineares Generalizados, incluindo uma estrutura de correlação entre as observações para a obtenção de estimativas sólidas e não viciadas. A função de quasiverossimilhança foi reexaminada por McCullagh e Nelder (1983). Sua grande vantagem é necessitar apenas da especificação da relação entre a média e a variância das observações, enquanto a verossimilhança necessita da especificação da forma de distribuição das observações (Agranonik, 2009).

Para um membro da família exponencial uniparamétrica, a função logverossimilhança é a mesma que a de quasi-verossimilhança. Para definir a função de quasi-verossimilhança, considera-se $y_{i}, i=1, \ldots, n$ observações 
independentes com médias $\mu_{i}$ e variâncias $V\left(\mu_{i}\right)$, sendo $V$ uma função conhecida. Supõe-se que cada observação $\mu_{i}$ seja uma função conhecida de um conjunto de parâmetros $\beta_{1}, \ldots, \beta_{p}$. Então para cada observação a função de quasiverossimilhança $\mathrm{Q}\left(\mathrm{y}_{\mathrm{i}} ; \mu_{\mathrm{i}}\right)$ é definida como:

$$
\frac{\partial \mathrm{Q}(\mathrm{yi} ; \mu \mathrm{i})}{\partial \mu \mathrm{i}}=\frac{\mathrm{yi}-\mu \mathrm{i}}{\mathrm{V}(\mu \mathrm{i})}
$$

Neste estudo, foi adotado a distribuição de probabilidade de Poisson e uma função de ligação canônica (logaritmo da média), com o preditor linear nit $=\operatorname{In}(\mu \mathrm{it})=\beta 0+\beta 1 \mathrm{X}$, cujo exponencial do parâmetro estimado representa 0 risco relativo (RR), em que $X$ representa a matriz de classificações dicotomizadas de precipitação, considerando 0 para ausência e 1 para a presença.

Segundo Agresti (1996) a definição do risco relativo é dada pela razão entre as probabilidades de sucesso de dois níveis da variável explicativa.

$E$ a fórmula do Risco Relativo é: $R R=\pi 1 / \pi 2$.

Todas as técnicas estatísticas mencionadas foram realizadas com 0 auxílio do software estatístico livre R 3.1.0. (R, 2008).

\section{RESULTADOS E DISCUSSÕES}

Os municípios em estudo foram agrupados em quatro regiões homogêneas (Anova), a partir de características semelhantes no regime de precipitação. O primeiro grupo (valor-p<0,05) foi denominado por região $A$, composto pelos municípios de Cruzeiro do Sul, Eirunepé, Rio Branco e Tarauacá. O segundo denominado por região $B($ valor- $p<0,05)$ com Labréa, Manicoré e Matupá, o terceiro por região $C$ (valor-p<0,05) sendo Altamira, Itaituba e Manaus, e o quarto por região $D($ valor- $<<0,05)$ composta por Belterra, Monte Alegre, Óbidos e Porto de Moz. Para comparação entre os totais pluviométricos e a sazonalidade da temperatura do ar nas regiões formadas utilizaram-se médias mensais do período de 2000 a 2015, normal climatologia para o período seco (1960 a 1990) divulgado pelo INMET (2016). A partir da Figura 2 é possível visualizar as regiões $A, B, C$ e D. 


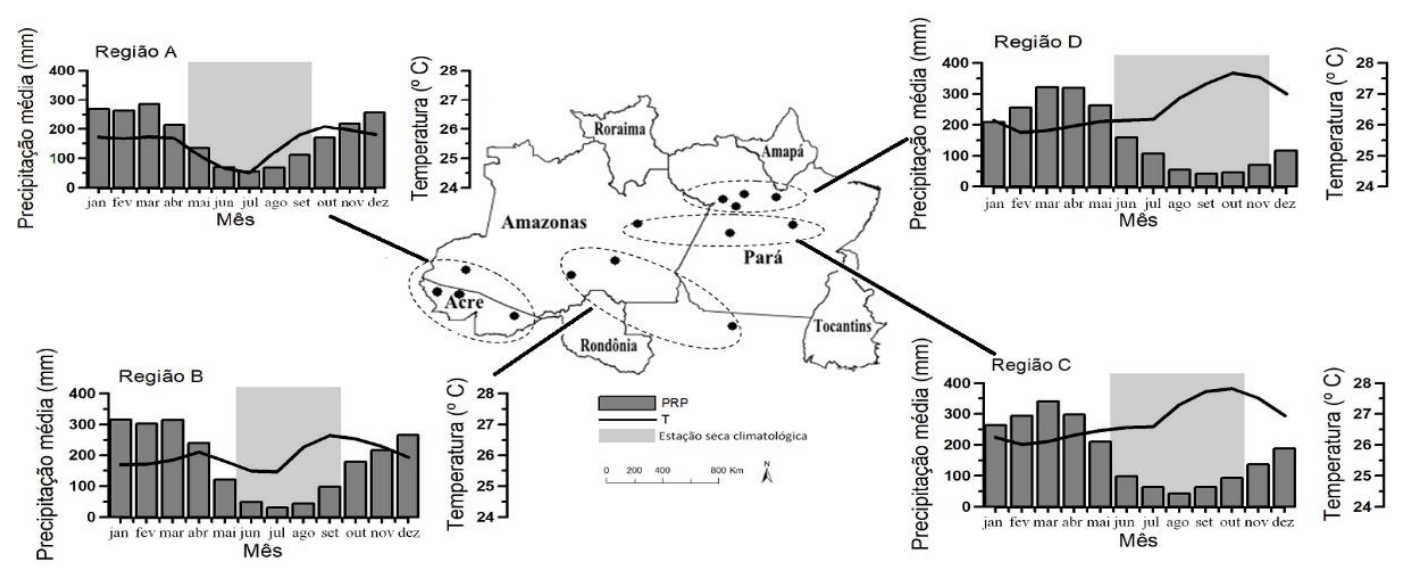

Figura 2 - Distribuição dos grupos denominados por regiões A, B, C e D.

A partir das médias mensais observadas para o período de 2000 a 2015, verifica-se que as regiões A e B apresentaram variabilidade sazonal semelhante, os meses de junho, julho e agosto caracterizaram-se o período seco. Na região C, o período seco ocorreu durante julho, agosto e setembro, apresentando um mês de defasagem em relação ao período das regiões $A$ e $B$. Os meses de agosto, setembro e outubro foram o período seco da região $D$, que apresentou um mês de defasagem em relação à região $C$ e dois em relação às regiões $A$ e $B$ (Figura 3).

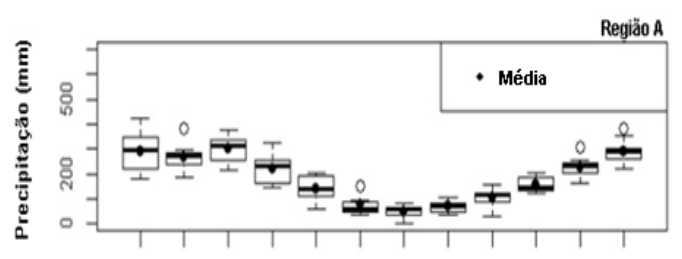

Jan Fev Mar Abr Mai Jun Jul Ago Set Out Nov Dez

Meses

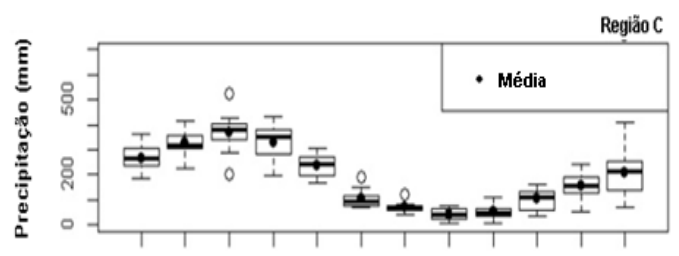

Jan Fev Mar Abr Mai Jun Jul Ago Set Out Nov Dez

Meses

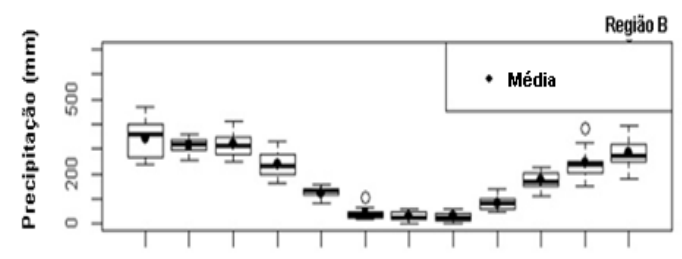

Jan Fev Mar Abr Mai Jun Jul Ago Set Out Nov Dez

Meses

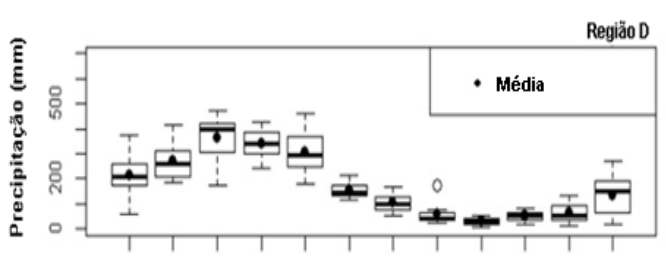

Jan Fev Mar Abr Mai Jun Jul Ago Set Out Nov Dez

Meses

Figura 3 - Boxplot de precipitação mensal do período de 2000 a 2015 das regiões A, B, C e D. 
Ao analisar as precipitações que ocorrem durante os anos de 2005, 2010 e 2015 na região A (Figura 4) constata-se que para os municípios de Cruzeiro do Sul, Eirunepé, Rio Branco e Tarauacá a seca do ano de 2005 foi a que mais influenciou o regime pluviométrico da região. Segundo Saatchi et al. (2013) a principal causa desse evento esteve relacionada ao comportamento médio da temperatura da superfície do mar (TSM) da bacia do Atlântico Tropical Norte. Foi possível observar também que em 2005 os meses mais secos ocorreram de maio a setembro, destacando o mês de julho dentro do período seco da série de 2000 a 2015.

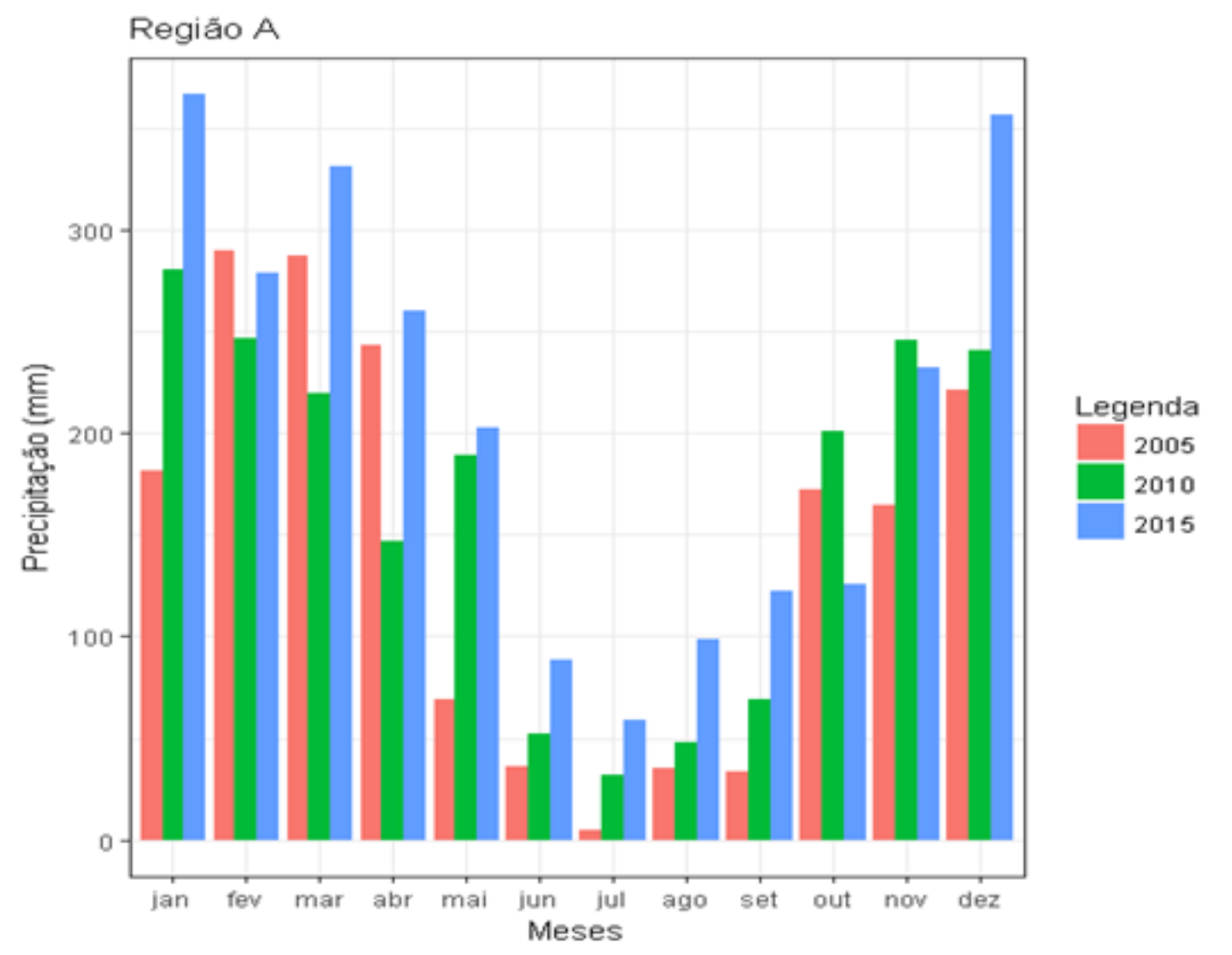

Figura 4 - Precipitação mensal dos anos 2005, 2010 e 2015 da região A.

Na Figura 5 constata-se que o regime pluviométrico durante os três eventos de seca na região $B$, os municípios de Labréa, Manicoré e Matupá, apresentaram período menos chuvoso entre os meses de maio a setembro. Destaca-se que ocorreram diferentes impactos dos eventos de seca nessa região. Ao observar o mês mais seco agosto, percebe-se que foi a seca de 2010 que mais influenciou o regime, já em relação aos meses de setembro a dezembro constata-se que a seca do ano de 2015 foi a mais relevante. 


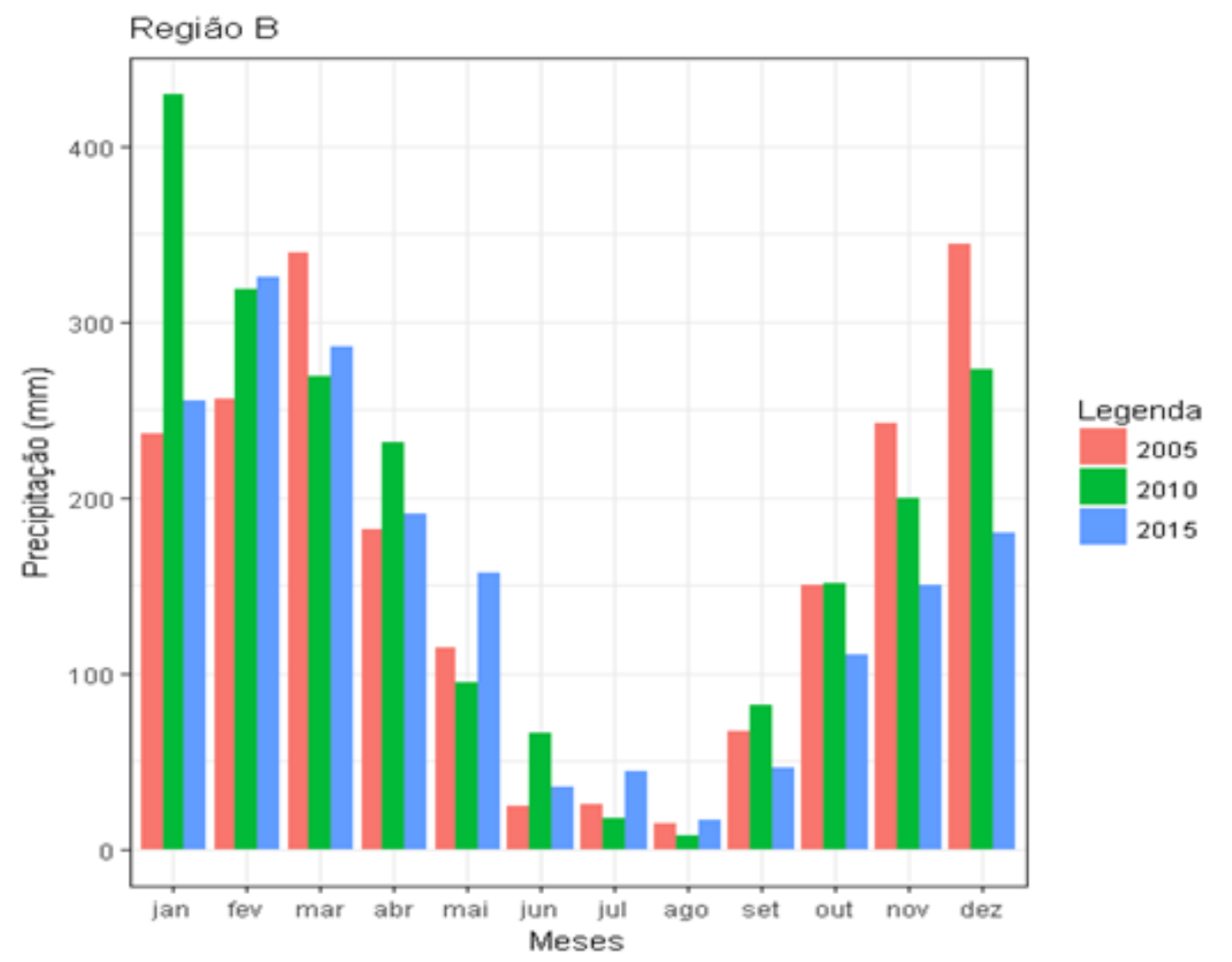

Figura 5 - Precipitação mensal dos anos 2005, 2010 e 2015 da região B.

$O$ regime pluviométrico da região $C$ também foi influenciado pela seca ocasionada pelos três eventos. Destacando o maior impacto ocorreu no ano de 2015, onde as médias mensais foram abaixo em relação aos anos de 2005 e 2010. É perceptível que no ano de 2015 ocorreu um prolongamento do período de estiagem deixando de ser de junho a outubro (Figura 3) para ocorrer de junho até dezembro. Ressalta-se ainda, o mês de janeiro de 2005, que apresenta discrepância inferior, quando comparado com as demais regiões (Figura 6). 


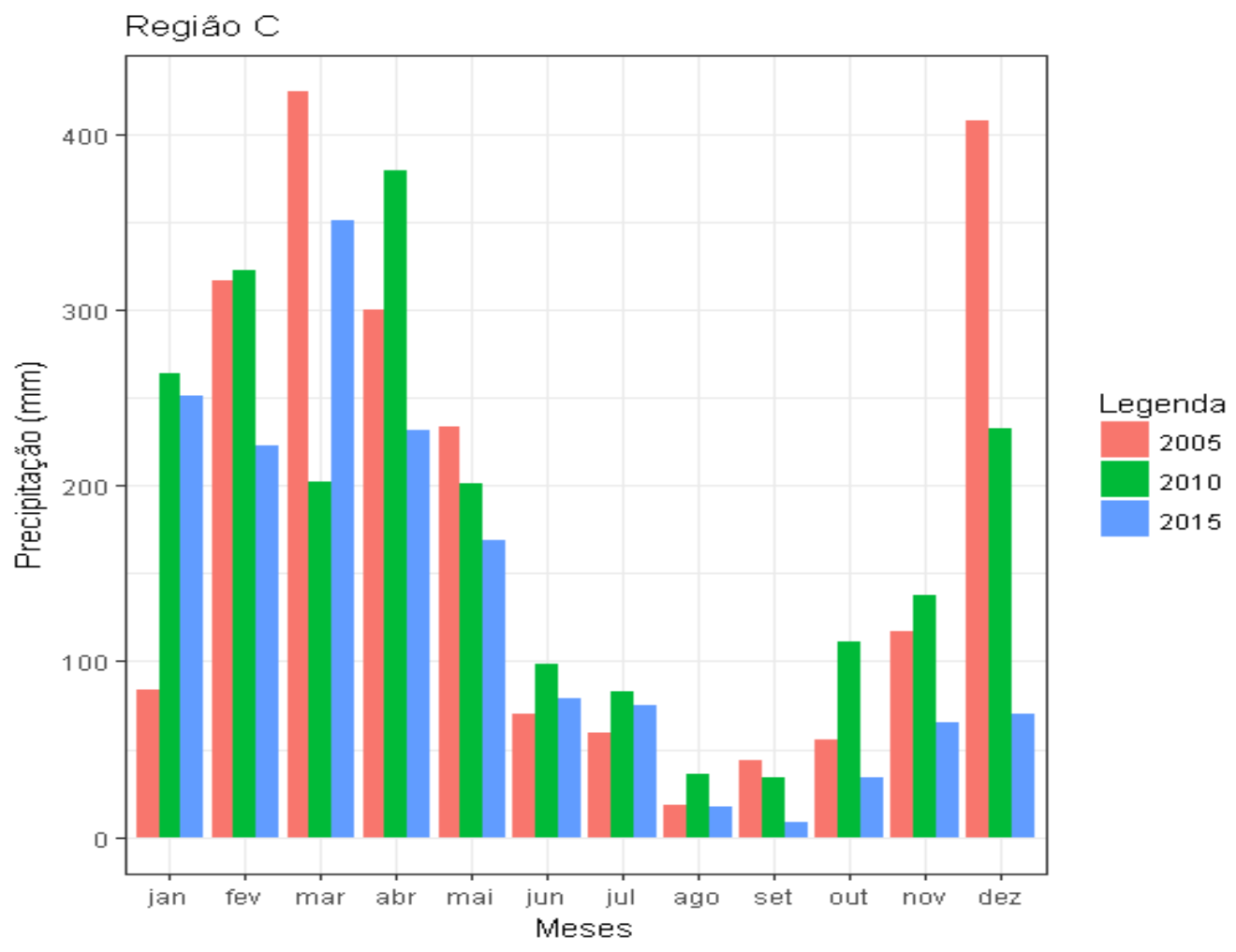

Figura 6 - Precipitação mensal dos anos 2005, 2010 e 2015 da região C.

Dentre as áreas em estudo a região $D$, foi a que apresentou maior alteração no regime pluviométrico, na Figura 7 é possível observar todos os três eventos de seca tiveram impacto para todos os municípios do Oeste do Pará. Como na região C no ano de 2005 o período de estiagem foi prolongado e no ano de 2015, não se observou período chuvoso para os municípios de Belterra, Monte Alegre, Óbidos e Porto de Moz, que normalmente se inicia no mês de novembro. 


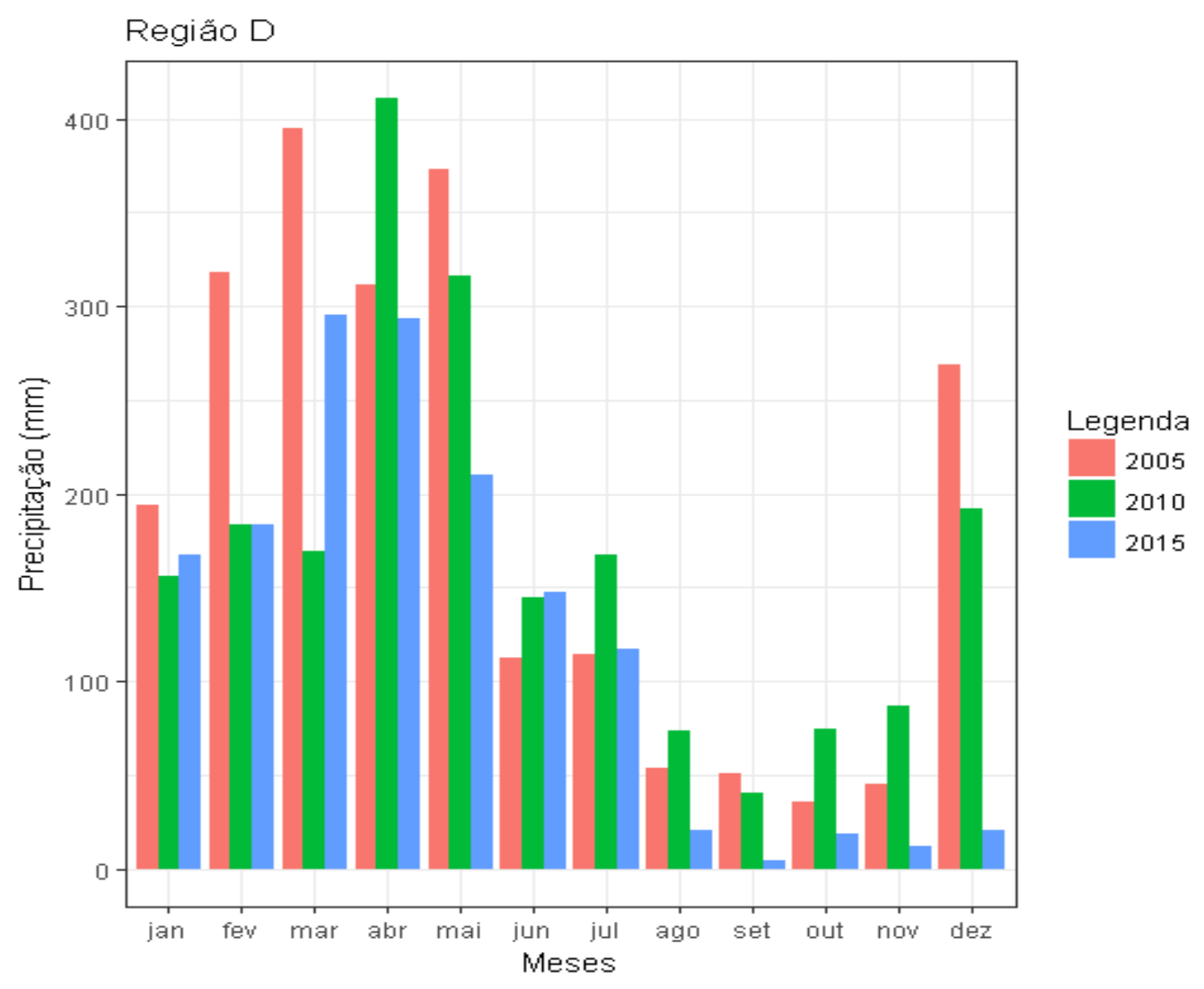

Figura 7 - Precipitação mensal dos anos 2005, 2010 e 2015 da região D.

Ao fazer comparação entre as regiões percebe-se que os três eventos de seca afetam de forma diferente os quatros grupos. O que está associado ao efeito diferencial dos mecanismos causadores das secas nas diferentes áreas da Amazônia (Saatchi et al, 2013). As estações que sofreram com a seca de 2005 (região A) localizada a sudoeste da Amazônia também foram pouco afetadas negativamente pelo evento de 2015 , tendo até mesmo aumento acima de 500 $\mathrm{mm}$ ano-1 nos seus totais pluviométricos em relação a normal climatológica, evidenciando o quão distintos foram os sistemas originadores destas secas. Embora o evento de seca de 2010 tenha sido mais extenso espacialmente do que 2005 (Lewis et al, 2011), o efeito da diminuição das chuvas nos dados observados de um evento pra outro só se mostrou perceptível nas estações da região $B$, mais ao centro da Amazônia. Já a seca de 2015 teve maior impacto nas estações das regiões C e D.

Os municípios amazônicos analisados quando sujeitos a períodos anomalamente secos, passam a ter maior probabilidade de ocorrência de queimadas que podem destruir centenas de milhares de hectares de floresta e injetar na atmosfera grandes quantidades de fumaça e aerossóis que poluem o ar em extensas áreas (Andreae et al., 2004), além de afetar a saúde das populações locais. Na Figura 8 observa-se a variabilidade dos números de internações por doenças nas quatro regiões em estudo para 2000-2015. As maiores médias do número de internações foram observadas no mês de junho 
nas regiões $A, C$ e $D$. É importante destaca-se a região $B$, sendo a que apresentou maior variabilidade e médias mais elevadas.

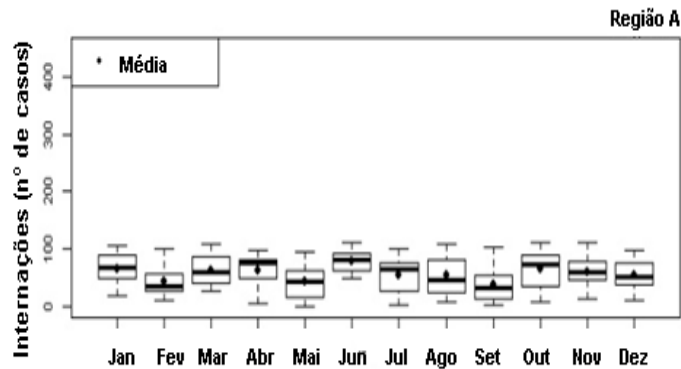

Meses

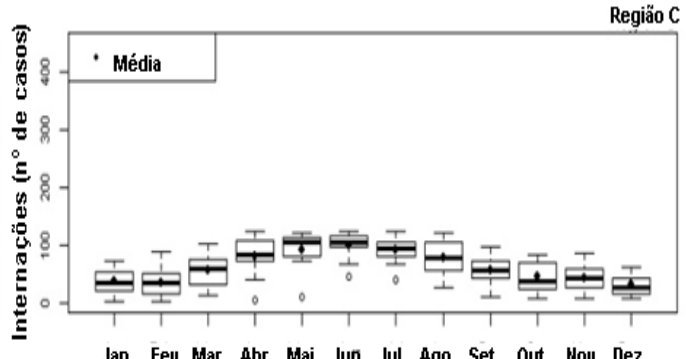

Meses

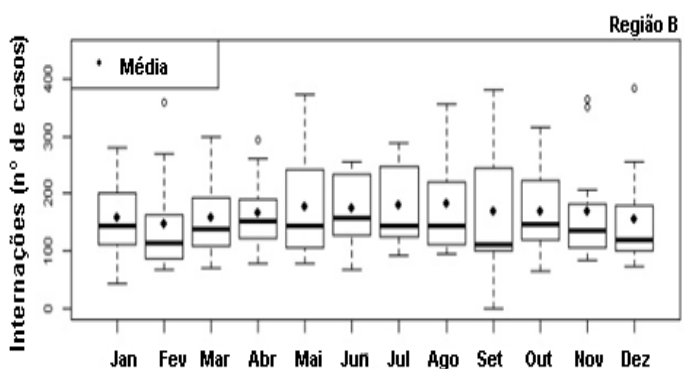

Meses

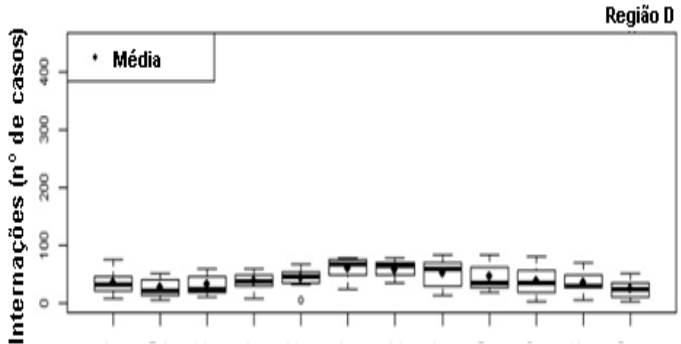

Jan Fev Mar Abr Mai Jun Jul Ago Set Out Hov Dez

Meses

Figura 8 - Médias de internações mensais por doenças respiratórias do período de 2000 a 2015 das regiões $A, B, C$ e D.

O modelo via Equações de Estimação Generalizada (GEE) utilizado para verificar associação e risco relativo (RR) entre as internações por causas respiratórias e a ausência de precipitação foi ajustado para cada região em estudo. A Tabela 1 evidência que para a região $A$, apenas a precipitação do município de Eirunepé não apresentou associação significativa (valor-p=0,242). Foram encontrados RR de 1,11, 1,09 e 1,05 na ausência de precipitação em Cruzeiro de Sul, Rio Branco e Tarauacá, respectivamente.

Tabela 1 - Modelo das Equações de Estimação Generalizada entre as internações por doenças respiratórias e ausência de precipitação na Região A no período de 2000 a 2015.

\begin{tabular}{cccccc}
\hline Municípios & Estimativas & Erro & Valor- p & RR & IC (95\%) \\
Cruzeiro do Sul & 0,1010 & 0,0374 & 0,007 & 1,11 & $1,03-1,19$ \\
Eirunepé & 0,0450 & 0,0385 & 0,242 & -- & -- \\
Rio Branco & 0,0926 & 0,0271 & 0,001 & 1,09 & $1,04-1,16$ \\
Tarauacá & 0,0876 & 0,0239 & 0,00025 & 1,05 & $1,04-1,14$ \\
\hline
\end{tabular}

*Significância a 5\%. *IC=Intervalo de Confiança.

A Tabela 2 é referente a região $B$, constata-se associação significativa entre as doenças respiratórias e a ausência de precipitação em Manicoré (valor- 
$p=0,008)$, com $R R$ de 1,10 . Dentro o grupo da região $B$, está o município de Matupá pertencente ao estado do Mato Grosso, que está localizado dentro do arco do desmatamento. Viana et al., (2010) afirmam que no período de seca, especialmente entre os meses de junho a setembro, ocorrem incrementos significativos de queimadas e doenças respiratórias em diversos municípios do estado de Mato Grosso. A combustão de biomassa gera a dispersão de diversos poluentes que comprovadamente causam uma série de efeitos sobre o ecossistema e à saúde humana, ocasionando expressivas taxas de internações por doenças respiratórias (Botelho et al., 2003; Peres et al., 2009; Silva et al., 2009; Silva et al., 2010).

Tabela 2 - Modelo das Equações de Estimação Generalizada entre as internações por doenças respiratórias e ausência de precipitação na Região B no período de 2000 a 2015.

\begin{tabular}{cccccc}
\hline Municípios & Estimativas & Erro & Valor- p & RR & IC (95\%) \\
Labréa & 0,000458 & 0,001380 & 0,740 & -- & -- \\
Manicoré & 0,0953 & 0,0357 & 0,008 & 1,10 & $1,03-1,18$ \\
Matupá & 0,0176 & 0,0480 & 0,714 & -- & -- \\
\hline
\end{tabular}

Significância a 5\%.

Para a região $\mathrm{C}$, foram captadas associações significativas entre as internações e a ausência de precipitação em Manaus (valor-p<0,001) e em Itaituba (valor- $p<0,001$ ). Em Manaus-AM, o RR encontrado sugere proteção e não fator de risco. Dentre os RR observados, ressalta-se o verificado no município de Itaituba - PA, onde a probabilidade da ocorrência de internações por causa respiratória é $130 \%$ maior quando não ocorre precipitação (Tabela 3).

Tabela 3 - Modelo das Equações de Estimação Generalizada entre as internações por doenças respiratórias e ausência de precipitação na Região C no período de 2000 a 2015.

\begin{tabular}{cccccc}
\hline Municípios & Estimativas & Erro & Valor- p & RR & IC (95\%) \\
Altamira & 0,331 & 0,260 & 0,2 & -- & -- \\
Manaus & $-1,311$ & 0,202 & $<0,001$ & 0,21 & $0,18-0,4$ \\
Itaituba & 2,590 & 0,285 & $<0,001$ & 13,33 & $7,62-23,3$ \\
\hline
\end{tabular}

Significância a 5\%.

Na região D (Tabela 4) constata-se ocorrência de associação significativa entre as internações e a ausência de precipitação com todos os municípios da região. Sendo o município de Monte Alegre-PA o que apresentou maior $R R$, $a$ probabilidade da ocorrência de internações por causa respiratória é $20,13 \%$ maior quando não ocorre precipitação.

Tabela 4 - Modelo das Equações de Estimação Generalizada entre as internações por doenças respiratórias e ausência de precipitação na Região D no período de 2000 a 2015.

\begin{tabular}{cccccc}
\hline Municípios & Estimativas & Erro & Valor- p & RR & IC (95\%) \\
Belterra & 0,3286 & 0,0627 & $<0,001$ & 1,39 & $1,23-1,57$ \\
Monte Alegre & 0,7583 & 0,0440 & $<0,001$ & 2,13 & $1,96-2,33$ \\
Óbidos & 0,4066 & 0,0421 & $<0,001$ & 1,50 & $1,38-1,63$ \\
Porto de Moz & 0,6916 & 0,0602 & $<0,001$ & 2,00 & $1,77-2,25$ \\
\hline
\end{tabular}

Significância a 5\%. 
Há muito as sociedades humanas vêm acumulando experiências com vicissitudes climáticas naturais segundo a Organização Mundial da Saúde WHO, (2008). Embora a saúde humana possa parecer estar relacionada primordialmente a um comportamento prudente, de hereditariedade, ocupação, exposições ao meio ambiente local e acesso a assistência médica, a saúde sustentada da população requer os "serviços vitais" da biosfera. As populações dependem de suprimentos de alimentos e água, de estar livres de excessos de doenças e do conforto físico propiciado pela estabilidade climática. Ainda segundo a WHO, (2008) de forma mais intensa, desastres e surtos de doenças frequentemente ocorrem em resposta aos ciclos climáticos regionais extremos, como, por exemplo, o ciclo de Oscilação Sul / El Niño (ENSO).

Eventos climáticos extremos podem ter impactos muito diferentes, como foi observado nos resultados expostos anteriormente, isto ocorre em razão de diferenças na capacidade da população alvo de lidar com a situação (McMichael, 2002). É preciso compreender que contra a ocorrência natural dos eventos climáticos pouco ou quase nada se pode fazer, no entanto a necessidade da resposta ao risco é de grande relevância. Diante deste cenário, o ideal é desenvolver ações para aumentar a resiliência ao que indiretamente reduz as ameaças. Com exceção das capitais, a maioria dos municípios Amazônicos é considerada comunidades vulneráveis diante desse novo cenário climático (IPCC, 2013).

Para uma melhor redução deste quadro Amazônico, deve-se considerar que os principais determinantes da capacidade de adaptação de uma comunidade são: riqueza econômica, informação, habilidades, infraestrutura, instituições. A capacidade de adaptação também é uma função do estado atual de saúde da população e da carga de doenças já existentes. Acredita-se que além da expansão cientifica em relação ao clima e a saúde na Amazônia, o acesso a informação passa a ser a principal resposta ao risco.

\section{CONCLUSÕES}

Conclui-se que os três eventos de seca afetam de forma diferente os municípios estudados, principalmente em relação ao risco na saúde acarretado pela ausência de chuva. Isto possivelmente está anexo a origem dos eventos nas diferentes áreas da Amazônia e na capacidade de cada população em enfrentar as adversidades ocasionadas pela seca.

A reflexão do artigo sinaliza para a necessidade de programar estratégias de adaptação direcionadas para proteger a saúde pública quer ou não sejam adotadas ações para mitigar os impactos dos eventos climáticos extremos. A capacitação é um passo preparatório essencial. A adaptação à estes eventos irá exigir mais do que recursos financeiros, tecnologia e infraestrutura em saúde pública. Educação e conscientização unidas a instituições proporcionará um ambiente que possibilite que as pessoas tomem decisões bem informadas e sustentáveis de longo prazo, são todos elementos necessários.

É importante ressaltar que uma das limitações do presente estudo foi não termos obtido informações de hábitos que podem influenciar na ocorrência das internações, para um melhor ajuste do modelo como, por exemplo, outros fatores de risco (tabagismo, obesidade, colesterol). 
Neste tipo de estudo apesar de não haver observações individuais, os resultados da modelagem estocástica evidenciam associações significativas apontando a existência de risco de forma coerente.

\section{AGRADECIMENTO}

Os autores agradecem a CAPES pela concessão da bolsa de pósdoutorado concedida à primeira autora (Projeto. 88881.064980/2014-01).

\section{REFERÊNCIAS}

AGRESTI, A. An Introduction to Categorical Data Analysis, New York: John Wiley \& Sons, Inc. 1996.

ANDREAE, M.O., et al., 2004. Smoking rain clouds over the Amazon. Science 303,1337-1342.

BOTELHO, C.; Correia, A. L.; Silva, A. M. C.; Macedo, A. G.; Silva, C. O. S. Fatores ambientais e hospitalizações em crianças menores de cinco anos com infecção respiratória aguda. Cad. Saúde Públ. 2003;19(6):1771-80.

COELHO, C. A. S.; Cavalcanti, I. A. F.; Costa, S. M. S.; Freitas, S. R.; Ito, E. R.; Luz, G.; Santos, A. F.; Nobre, C. A.; Marengo, J. A.; Pezza, A. B. 2012. Climate diagnostics of three major drought events in the Amazon and illustrations of their seasonal precipitation predictions. Meteorological Applications 19, 237-255. DOI: $10.1002 /$ met.1324.

COUGHLAN, M. J. 1987. Monitoring drought in Australia. In D. A. Wilhite and W. E. Easterling, eds. Planning for Drought: Toward a Reduction of Societal Vulnerability; Chapter 10. Westview Press, Boulder, Colorado, U.S.A.

Departamento de Informática do Sistema único de Saúde - DATASUS, 2016. Informações de Saúde (TABNET), Morbidade Hospitalar do SUS (SIH/SUS). Disponível em http://www2.datasus.gov.br/DATASUS/index.php?area $=0203 \& i d=6926$. Acesso em: 05 jun. 2016.

DE SOUZA, E.B. et al. On the influences of the El Niño, La Niña and Atlantic dipole pattern on the Amazonian rainfall during 1960-1998. ActaAmazonica, $v$. 30, n. 2, p. 305-318, 2000.

DE SOUZA, E. B., M. T. Kayano, and T. Ambrizzi, 2005: Intraseasonal and submonthly variability over the eastern Amazon and northeast Brazil during the autumn rainy season. Theor. Appl. Climatol., 81, 177-191, doi:10.1007/s00704-004-0081-4.

INTERGOVERNMENTAL PANEL ON CLIMATE CHANGE (IPCC). Climate change 2007: the physical science basis. Contribution of working group I to the fourth assessment report of the intergovernmental panel on climate change. Cambridge: Cambridge University, 2007. 996p. Disponível em: <https://www.ipcc-wg1.unibe.ch/publications/wg1-ar4/wg1-ar4.html>. Acesso em: 29 de mai. de 2016.

INTERGOVERNMENTAL PANEL ON CLIMATE CHANGE (IPCC). Summary for Policymakers. In: Climate Change 2013: The Physical Science Basis. 
Contribution of Working Group I to the Fifth Assessment Report of the Intergovernmental Panel on Climate Change [Stocker, T.F. et al. (eds.)]. Cambridge: Cambridge University, 2013. 33p. Disponível em: <http://www.climatechange2013.org/images/report/WG1AR5_SPM_FINAL.pdf>. Acesso em: 10 jun. 2016.

INSTITUTO NACIONAL DE PESQUISAS ESPACIAIS - INPE, 2016. Portal do Monitoramento de Queimadas e Incêndios. Disponível em http://www.inpe.br/queimadas. Acesso em: 19 de jun. de 2016

MCKEE, T.B.N., J. DOESKEN, AND J. KLEIST, 1993: The relationship of drought frecuency and duration to time scales. Eight Conf. On Applied Climatology. Anaheim, CA, Amer. Meteor. Soc. 179-184.

INSTITUTO NACIONAL DE METEOROLOGIA - INMET, 2016. Banco de Dados Meteorológicos para Ensino e Pesquisa - BDMEP. Disponível em http://www.inmet.gov.br/projetos/rede/pesquisa/. Acesso: 12/04/2016.

LEVENE, H. (1960). Robust testes for equality of variances. In Contributions to Probability and Statistics (I. Olkin, ed.) 278-292. Stanford Univ. Press, Palo Alto, CA.

LEWIS, S. L.; BRANDO, P. M.; PHILLIPS, O. L.; VAN DER HEIJDEN, G. M. F.; NEPSTAD, D. The 2010 Amazon Drought. Science, v. 331, n. 6017, p. 554, 2011.

LIANG, K. Y.; ZEGER, S. L. Longitudinal data analysis using generalized linear models. Biometrika. 73:13-22. 1986.

MARENGO, J.A.; NOBRE, C.A.; TOMASELLA. J.; OYAMA, M.D.; SAMPAIO, G.; CAMARGO, H.; ALVES, L.M. 2008. The drought of Amazonia in 2005. Journal of Climate, 21: 495-516.

MARENGO, J. A.; NOBRE, C. A.; TOMASELlA, J.; CARDOSO, M. F.; OYAMA, M. D. 2008a. Hydroclimatic and ecological behaviour of the drought of Amazonia in 2005. Philosophical Transactions of the Royal Society of London 363: 17731778. DOI: $10.1098 /$ rstb.2007.0015.

MARENGO, J. A.; NOBRE, C. A.; TOMASELLA, J.; OYAMA, M. D.; OLIVEIRA, G. S.; OLIVEIRA, R.; CAMARGO, H.; ALVES, L. M.; BROWN, F. 2008b. The drought of Amazonia in 2005. Journal of Climate 21, 495-516. DOI: 10.1175/2007JCLI1600.1.

MARENGO JA, ET AL. The drought of 2010 in the context of historical droughts in the Amazon region. Geophys Res Lett. 2011;38:L12703. doi: 10.1029/2011GL047436.

MCMICHAEL, A.J. Population, environment, disease, and survival: past patterns, uncertain futures. Lancet, 359: 1145-48 (2002).

MOLD. Una breve guía para elmoholahumedad y suhogar. Agency. [site na internet]. Washington, DC, United States Environmental Protection; 2008. http://www.epa.gov/mold/moldresources.html. Acesso: 18/04/2016.

MOURÃO DS, VIANA L, HACON S, BARCELLOS C. Impacto das emissões de queimadas para a saúde em duas áreas do Estado de Mato Grosso - Amazônia Legal. Cientifica, Rio de Janeiro, 2007. 
NOBRE, P.; SHUKLA J. Variations of sea surface temperature, wind stress, and rainfall over the tropical Atlantic and South America. J. Climate, v.9, n.19, p.2464-2479, 1996.

PAN, W. Information criterion in generalized estimating equations. Biometrics. 57(1): 120-125, 2001.

PERES, W. L.; OLIVEIRA, R.C; VIANA, D. V.; SANTOS, W. S. Epidemiologia da asma em crianças menores de 5 anos de idade em Cuiabá/MT. J BrasPneumol. 2009;35 Supl 1R: R1-R40.

ROSA AM, IGNOTTI E, HACON SS, CASTRO HA. Análise das internações por doenças respiratórias em Tangará da Serra - Amazônia Brasileira. J BrasPneumol. 2008a;34:575-82.

ROSA AM, IGNOTTI E, BOTELHO C, CASTRO HA, HACON SS. Doença respiratória e sazonalidade climática em menores de 15 anos em um município da Amazônia brasileira. J Pediatr (Rio J). 2008b; 84(6):543-9.

SAATCHI, S.; ASEFI-NAJAFABADY, S.; MALHI, Y.; ARAGÃO, L. E. O. C.; ANDERSON, L. O.; MYENI, R. B.; NEMANI, R. Persistent effects of a severe drought on Amazonian forest canopy. Proceedings of the National Academy of Sciences. v.110 n. 2 p.565-570, 2013.

SANTOS, C. A. C. DOS; SATYAMURTY, P; SANTOS, E. M. dos. 2012. Tendências de índices de extremos climáticos para a região de Manaus-AM. Acta Amazonic 42, 329-336.

SANTOS, E. B.; LUCIO, P. S.; SANTOS E SILVA, C. M. 2014. Precipitation regionalization of the Brazilian Amazon. Atmospheric Science Letters. DOI: $10.1002 /$ asl2.535.

SANTOS, E. B., P. S. LUCIO, AND C. M. SANTOS E SILVA, 2015: Precipitation regionalization of the Brazilian Amazon. Atmos. Sci. Lett., doi:10.1002/asl2.535, in press.

SENA, J. A.; BeSER DE DEUS, L. A.; FREITAS, M. A. V.; COSTA, L. 2012. Extreme events of droughts and floods in Amazonia: 2005 and 2009, Water Resources Management 26, 1665- 1676. DOI: 10.1007/s11260-012-9978-3.

SILVA, A. M. C.; MATTOS, I. E.; FREITAS, S.R.; LONGO, K. M.; HACON, S. S. Material particulado (PM2.5) de queima de biomassa e doenças respiratórias no sul da Amazônia brasileira. Rev. Bras. Epidemiol. 2010; 13(2): 337-51.

SOLÉ D, WANDALSEN GF, CAMELO-NUNES IC, NASPITZ CK; ISAAC - Grupo Brasileiro. Prevalence of symptoms of asthma, rhinitis, and atopic eczema among Brazilian children and adolescents identified by the International Study of Asthma and Allergies in Childhood (ISAAC) - Phase 3. J Pediatr (Rio J). 2006;82(5):341-6. DOI:10.1590/S0021-75572006000600006

THOMAZELLI LM, VIEIRA S, LEAL AL, SOUSA TS, OLIVEIRA DB, GOLONO MA, ET $A L$. Surveillance of eight respiratory viruses in clinical samples of pediatric patients in southeast Brazil. J Pediatr (Rio J). 2007;83:422-8.

VALE, R.; FILIZOLA, N.; SOUZA, R.; SCHONGART, J. 2011. A cheia de 2009 na Amazônia Brasileira. RevistaBrasileira de Geociências [online]. 41, 577-586. ISSN 0375-7536. 
WILHITE, D. A. 1993. Preparing for Drought: A Guidebook for Developing Countries. United Nations Environment Program, Nairobi, Kenya (in press).

ZEGER, S. L.; LIANG, K. Y. Longitudinal data analysis for discrete and continuous outcomes. Biometrics. 42:1(3)121-130. 1986. 